\title{
A pictorial key and diagnosis of the Brazilian genera of Micropezidae (Diptera, Nerioidea)
}

\author{
Gustavo Borges Ferro ${ }^{1} \&$ Claudio J. B. de Carvalho ${ }^{2}$
}

\author{
${ }^{1}$ Programa de Pós-graduação em Entomologia, Universidade Federal do Paraná, Caixa Postal 19020, 81531-980 Curitiba-PR, Brasil. \\ gustavo_ferro01@yahoo.com.br \\ ${ }^{2}$ Departamento de Zoologia, Universidade Federal do Paraná, Caixa Postal 19020, 81531-980 Curitiba-PR, Brasil. cjbcarva@ufpr.br
}

\begin{abstract}
A pictorial key and diagnosis of the Brazilian genera of Micropezidae (Diptera, Nerioidea). This paper provides the first pictorial key and diagnosis for the Brazilian genera of the Micropezidae, based on external morphological characters illustrated with photographs. The key includes 13 genera: Cardiacephala Macquart, Cliobata Enderlein, Grallipeza Rondani, Metasphen Frey, Micropeza Meigen, Parasphen Enderlein, Planipeza Marshall, Plocoscelus Enderlein, Poecilotylus Hennig, Ptilosphen Enderlein, Rainieria Rondani, Scipopus Enderlein and Taeniaptera Macquart. For each genus, the species known to occur in Brazil are listed and their distribution records, including new ones, are provided.
\end{abstract}

KEYWORDS. Brazil; Insecta; Neotropical; stilt-legged flies; taxonomy.

The Micropezidae comprise a cosmopolitan family of flies with the greatest species richness found in tropical regions (Steyskal 1968). The ca. 700 species in ca. 60 genera are distributed among five subfamilies: Calycopteriginae, Calobatinae, Eurybatinae, Micropezinae and Taeniapterinae (Marshall 2012), the last two of which are found in Brazil. While the Brazilian species diversity has not yet been fully examined, we do know that at least 273 species in 20 genera are found in the Neotropical region (Amorim et al. 2002). Adults are predominantly saprophagous and characteristically have long and slender legs, with anterior legs shorter, veins $\mathrm{R}_{4+5}$ and $\mathrm{M}$ converging (sometimes fused and pedunculated) at the wing apex, lacking both ocellar setae and vibrissae (Marshall 2010). Most species are similar in appearance to wasps and ants, especially species in the subfamily Taeniapterinae (Marshall 2012).

While larvae have been collected in banana trees and decaying wood, there are relatively few studies that examined immature forms (Fischer 1932; Steyskal 1964; Albuquerque 1972). Predatory micropezids are restricted to the subfamily Calobatinae, but they were never recorded in Brazil (Marshall 2012).

American species of the family were catalogued by Steyskal (1968). While these flies are relatively common, identification of the species in the Micropezidae is problematic and many genera are still in need of revision. Studies developed by Hennig (1934, 1935a, 1935b, 1936, 1937a, $1937 b, 1937 \mathrm{c})$, in the first half of the twentieth century, are still very important for the identification of most of the Neotropical species. Identification of some Argentinean species is possible with the keys provided by Aczél (1949, 1951). Marshall (2010), in the Manual of Central American Diptera, provides a key to the Neotropical genera. However, no such tool yet exists for the Brazilian genera in the Micropezidae.
Here we present, for the first time, a pictorial key and diagnosis of the Brazilian genera of Micropezidae.

\section{MATERIAL AND METHODS}

Specimens from the following collections (followed by curators in parenthesis) were analyzed: Coleção de Entomologia Pe. Jesus Santiago Moure, Departamento de Zoologia, Universidade Federal do Paraná, Curitiba, Paraná, Brasil, DZUP (Claudio J. B. de Carvalho); Museu de Zoologia da Universidade de São Paulo, São Paulo, Brasil, MZUSP (Carlos J. E. Lamas) and Instituto Nacional de Pesquisas da Amazônia, Manaus, Amazonas, Brasil, INPA (Márcio Luiz de Oliveira and José Albertino Rafael). Junior synonyms of species in Systema Dipterorum (2013) were accepted. New distribution records are indicated by an asterisk.

Pinned specimens were examined under a Zeiss Stemi DV4 stereomicroscope. Morphological terminology follows McAlpine (1981), except head characteristics, which followed Cresson (1930). Photographs were taken by a Leica MZ16 stereomicroscope and Leica DFC 500 camera, made available by the TAXon Line Project from DZUP.

\section{TAXONOMY}

\section{Pictorial key to Brazilian genera (modified from Marshall 2010)}

1. Katepisternum with no more than four setae (Fig. 1); bm-cu absent; frontal setae absent... Micropezinae ..... Micropeza Meigen ....................................................................... 2 - Katepisternum with numerous setae aligned vertically (Fig. 2); bm-cu present; frontal setae present... Taeniapterinae...3 
2. Lunule not visible (Fig. 3); single notopleural seta present . Micropeza (Micropeza) Meigen

- Lunule visible (Fig. 4); two notopleural setae present ..... Micropeza (Neriocephalus) Enderlein

3. Scutellum upturned (Fig. 5); arista pectinate, hairs on only one side (Fig. 7); mid and hind femur with a strong protuberance distally (Fig. 10)

- Scutellum not upturned (Fig. 6); arista bare (Fig. 8) or plumose (Fig. 9), sometimes hairs are small and do not exceed the basal half; mid and hind femur lacking strong protuberance (Fig. 11)

\section{5}

4. Paracephala swollen, no clear divisions between epicephala, giving the head a heart shape in dorsal view (Fig. $12)$; wing with round hyaline spots in the apical portion (Fig. 15) Cardiacephala Macquart

- Paracephala usually not swollen (Fig. 13), when swollen, with inner-verticals setae in conspicuous protuberances (Fig. 14); wing without hyaline rounded spots (Fig. 16) Plocoscelus Enderlein

5. Cell cup long, $\mathrm{CuA}_{2}$ vein always longer than $\mathrm{A}_{1}+\mathrm{CuA}_{2}$ (Fig. 17)

- Cell cup short, $\mathrm{CuA}_{2}$ vein always shorter than $\mathrm{A}_{1}+\mathrm{Cu} \mathrm{A}_{2}$ (Fig. 18)

6. Arista plumose, hairs present on both sides (Fig. 9) ..... 7

- Arista bare (Fig. 8) ......................................................... 8

7. Dorsocentral setae present (Fig. 19); mesofrons and epicephala clearly delimited ......... Ptilosphen Enderlein

- Dorsocentral setae sometimes absent; mesofrons and epicephala not clearly delimited ..... Parasphen Enderlein

8. Palpus enlarged basally, tapering to the apex (Fig. 20) ... .. Taeniaptera Macquart

- Palpus not enlarged, parallel sided with a rounded apex (Fig. 21) Poecilotylus Hennig

9. Arista bare (Fig. 8) 10

- Arista plumose (Fig. 9), sometimes hairs are small and do not exceed the basal half 13

10. Clypeus narrow (Fig. 22); apexes of $\mathrm{Sc}$ and $\mathrm{R}_{1}$ separated by no more than the width of $\mathrm{R}_{1}$ (Fig. 24)

Metasphen Frey

- Clypeus wide (Fig. 23); apexes of Sc and $\mathrm{R}_{1}$ separated by more than the width of $\mathrm{R}_{1}$ (Fig. 25) 11

11. Mesofrons with no clear divisions between epicephala (Fig. 26); head orange in most cases Scipopus Enderlein

- Mesofrons with conspicuous division with epicephala (Fig. 27); head color normally darkened 12

12. Length of Costa between $\mathrm{R}_{2+3}$ and $\mathrm{R}_{4+5}$ less than one-half as long as $\mathrm{M}$ after dm-cu (Fig. 28); palpus not enlarged, thin and spatulated (Fig. 30) .............. Cliobata Enderlein

- Length of Costa between $\mathrm{R}_{2+3}$ and $\mathrm{R}_{4+5}$ more than one-half as long as $\mathrm{M}$ after dm-cu (Fig. 29); palpus quite enlarged (Fig. 31) Rainieria Rondani
13. Arista always with hairs that extend past basal third; wing mostly dark brown with round hyaline spots (Fig. 32); thorax with anterior portion elongated, conspicuously surpassing the forecoxa (Fig. 33) ..... Planipeza Marshall

- Arista in some cases with small hairs that do not extend past basal third; wing hyaline, sometimes with darkened spots; thorax compact (Fig. 34) ........ Grallipeza Rondani

\section{Cardiacephala Macquart, 1843}

(Figs. 12, 15)

Cardiacephala Macquart, 1843: 399. Type species: Musca longipes Fabricius, 1787

Diagnosis. Dorsoventrally flattened head. Paracephala swollen, no clear divisions between epicephala, giving the head a heart shape in dorsal view (Fig. 12). Wing strongly infuscated or not, but always with rounded hyaline spots on the apical portion (Fig. 15).

Comments. This genus is mainly characterized by the protuberances in the paracephala. The similarity of Cardiacephala and Plocoscelus was discussed by Cresson (1930) and Hennig (1935b).

References. Cresson (1930), Hennig (1935b).

Brazilian species. Cardiacephala guttata (Walker, 1852) [Brazil (type-locality); Amazonas, Careiro*; Amazonas, Manaus, Reserva Ducke*; Bahia, Salvador (Albuquerque 1989); Ceará, Russas (Albuquerque 1989); Pará, Belém, Mocambo (Albuquerque 1989)], Cardiacephala secunda Hennig, 1935 [Brazil, Espírito Santo, Baixo Guandu*].

\section{Cliobata Enderlein, 1923}

(Figs. 27, 28, 30)

Cliobata Enderlein, 1923: 540. Type species: Calobata guttipennis Wiedemann, 1830.

Diagnosis. Larger and always darker species. Divisions between mesofrons and epicephala visible (Fig. 27). Palpus not enlarged, thin and spatulate (Fig. 30). Length of Costa between $\mathrm{R}_{2+3}$ and $\mathrm{R}_{4+5}$ less than one-half as long as $\mathrm{M}$ after dm-cu (Fig. 28). Peduncle of CuP vein visible in cup cell. Infuscated wing, sometimes with rounded hyaline spots.

Comments. Cliobata is a Neotropical genus, with most of the described species recorded from Brazil. Despite similarity with Scipopus in size and color, the divisions of the mesofrons and epicephala in Cliobata can easily be distinguished (Fig. 27), as Cresson (1930) and Hennig (1935b) indicated.

References. Cresson (1930), Hennig (1935b).

Brazilian species. Cliobata diadema (Wiedemann, 1830) [Brazil (type-locality); Rio de Janeiro, Corcovado*], Cliobata diademoides Hendel, 1933 [Brazil, Bahia (type-locality)], Cliobata fenestrata (Czerny, 1932) [Brazil, São Paulo, Alto da Serra (type-locality); Rio de Janeiro, Petrópolis*; Santa Catarina, Seara, Nova Teutônia*], Cliobata guttipennis (Wiedemann, 1830) [Brazil (type-locality)], Cliobata 

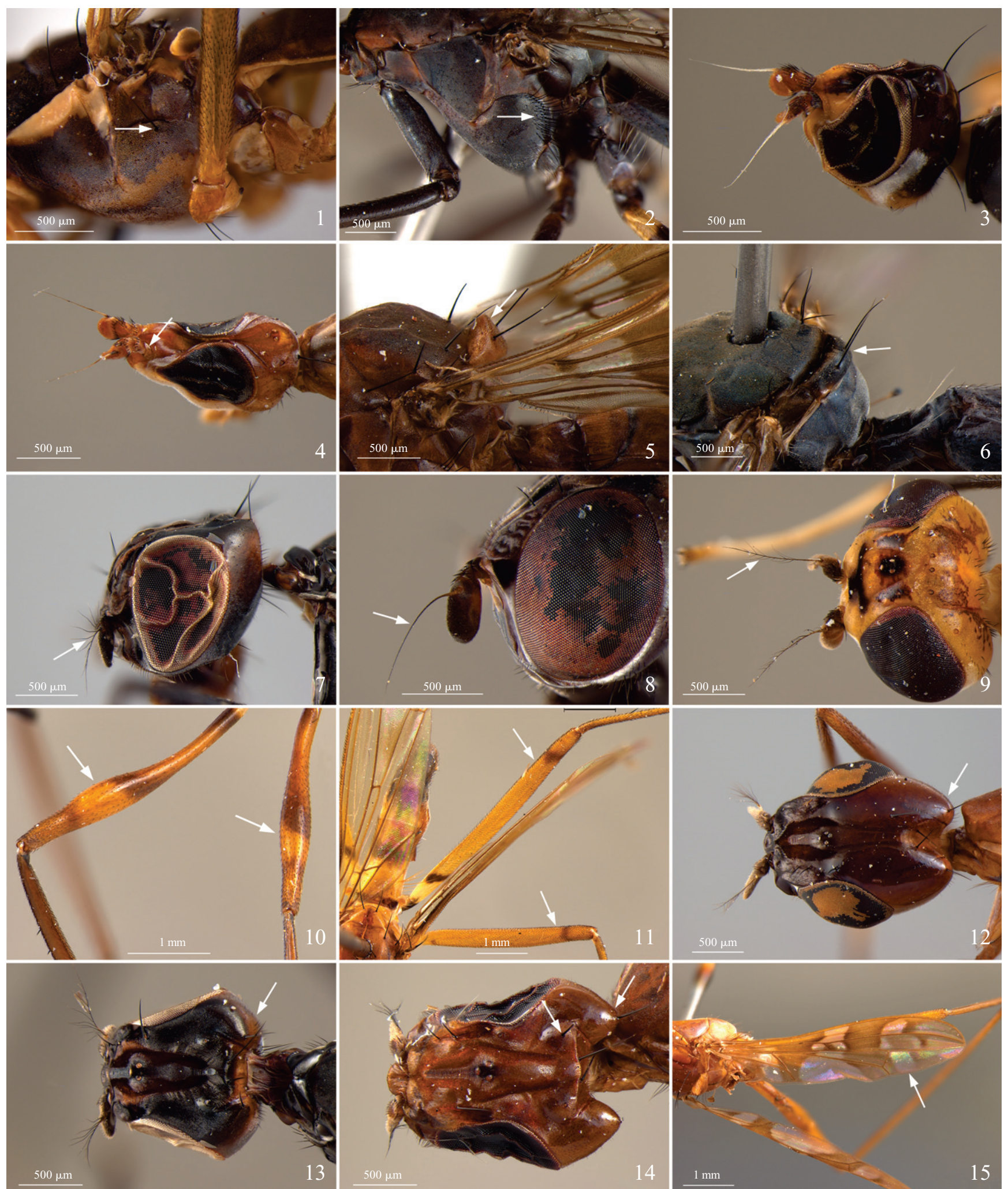

Figs. 1-15. 1. Thorax, lateral view, Micropeza sp. 1. (arrow: katepisternal seta); 2. Thorax, lateral view, Taeniaptera angulata (arrow: katepisternal setae); 3. Head, dorso-lateral view, Micropeza sp. 1.; 4. Head, dorso-lateral view, Micropeza (Neriocephalus) stigmatica (arrow: lunule); 5. Thorax, lateral view, Plocoscelus conifer (arrow: scutellum); 6. Thorax, lateral view, Taeniaptera angulata (arrow: scutellum); 7. Head, lateral view, Plocoscelus brevipennis (arrow: arista); 8. Head, lateral view, Taeniaptera angulata (arrow: arista); 9. Head, dorsal view, Grallipeza nigrinotata(arrow: arista); 10. Mid and hind femur, dorso-lateral view, Plocoscelus conifer (arrows: apical portion of femora); 11. Mid and hind femur, dorso-lateral view, Grallipeza nigrinotata (arrows: apical portion of femora); 12. Head, dorsal view, Cardiacephala gutatta (arrow: paracephala); 13. Head, dorsal view, Plocoscelusbrevipennis (arrow: paracephala); 14. Head, dorsal view, Plocoscelus conifer (arrows: paracephala and inner-vertical seta); 15. Wing, dorsal view, Cardiacephala gutatta (arrow: hyaline rounds). 
guttulipennis (Enderlein, 1922) [Brazil, Santa Catarina, Blumenau (type-locality); Paraná, Ponta Grossa*], Cliobata lunaris (Wiedemann, 1830) [Brazil (type-locality); Espírito Santo, Conceição da Barra*], Cliobata muelleri (Enderlein, 1922) [Brazil, Santa Catarina, Blumenau (type-locality)], Cliobata orcina (Wiedemann, 1830) [Brazil (type-locality)], Cliobata raptimanus (Bezzi, 1924) [Brazil, São Paulo (typelocality); Paraná, Piraquara, Mananciais da Serra*; Santa Catarina, São Bento do Sul*], Cliobata trimaculata (Czerny, 1932) [Brazil, Santa Catarina; São Paulo, São Vicente, Santos; Rio Grande do Sul, Santa Cruz (type-locality)].

\section{Grallipeza Rondani, 1850}

(Figs. 9, 11, 34)

Grallipeza Rondani, 1850: 180. Type species: Calobata unimaculata Macquart, 1846.

Diagnosis. Ground colour yellow to reddish brown. Arista plumose (Fig. 9), in some cases with small hairs that do not exceed the basal half. Thorax compact (Fig. 34). Hyaline wing, sometimes with darkened spots.

Comments. Grallipeza is recognized mainly by the short cell cup (Fig. 18) and plumose arista (Fig. 9), differing from Planipeza by the compact thorax (Fig. 34).

References. Cresson (1930), Hennig (1934).

Brazilian species. Grallipeza acutivitta (Hendel, 1936) [Brazil, Pará, Taperinha (type-locality)], Grallipeza affinis Hennig, 1934 [Brazil, Rondônia, Porto Velho, Área Mutum M7P3*; Roraima, Ilha de Maracá (Albuquerque 1991); Rondônia, Pacaraima (Albuquerque 1991)], Grallipeza amazonica (Enderlein, 1922) [Brazil, Amazônia (type-locality)], Grallipeza cantata (Cresson, 1926) [Brazil, "Rio Tefé, Boa Vista do Jaquiri” (type-locality)], Grallipeza hyaloptera (Hendel, 1936) [Brazil, Pará, Belém (type-locality)], Grallipeza imbecilla (Enderlein, 1922) [Brazil, Santa Catarina, Blumenau (type-locality)], Grallipeza nigrinotata Hennig, 1934 [Brazil, Amazonas, AM010 km31 Embrapa; Mato Grosso, Chapada dos Guimarães*; Pará, Oriximiná*; Rio de Janeiro, Gávea*; Rondônia, Cacaulândia, Sítio do João Cabeça*; Rondônia, Porto Velho, AHE Jirau Rio Madeira*; Rondônia, Vilhena*; São Paulo, Araçatuba, Córrego Azul*; São Paulo, Ribeirão Preto*], Grallipeza ornatithorax (Enderlein, 1922) [Brazil, Minas Gerais, Arceburgo*; Rio de Janeiro, Itatiaia*; Paraná, Curitiba*; Santa Catarina, Nova Teutônia, Seara*; São Paulo, Bocaina; São Paulo, Campos do Jordão*; São Paulo, Peruíbe*; São Paulo, São Paulo, Butantan*; São Paulo, Serra da Cantareira*], Grallipeza placidoides (Cresson, 1926) [Brazil, Roraima, Pacaraima (Albuquerque 1991); Maranhão, Rosário, Igarapé Paraqueú*], Grallipeza scurra (Enderlein, 1922) [Brazil, Amazonas, Manaus, Reserva Ducke*], Grallipeza simplex (Wiedemann, 1830) [Brazil, Mato Grosso, Chapada dos Guimarães*], Grallipeza suavis (Enderlein, 1922) [Brazil, Pará, Ilha do Marajó (type-locality)], Grallipeza unimaculata (Macquart, 1846) [Brazil].

\section{Metasphen Frey, 1927}

(Figs. 22, 24)

Metasphen Frey, 1927: 71. Type species: Metasphen vigilans Frey, 1927.

Diagnosis. Slender species, ground colour yellow to light brown. Palpus flattened and spatulate. Clypeus narrow, almost hidden in frontal view (Fig. 22); apexes of Sc and $\mathrm{R}_{1}$ separated by width of $\mathrm{R}_{1}$ at most (Fig. 24).

Comments. Marshall (2004) transferred Rainieria obscura Hennig, 1935, the type-species of Globomyia, a subgenus of Rainieria, to Metasphen.

References. Frey (1927), Hennig (1935b), Marshall (2004).

Brazilian species. Metasphen vigilans Frey, 1927 [Brazil, Amazonas, Manaus, Reserva Ducke*; Amazonas, Taracuá, Rio Uaupés (type-locality); Rondônia, Cacaulândia, Sítio do João Cabeça*].

\section{Micropeza Meigen, 1803}

(Figs. 1, 3, 4)

Micropeza Meigen, 1803: 276. Type species: Musca corrigiolata Linnaeus, 1767.

Diagnosis. Head tapered, sometimes much longer than wide (Fig. 4) or less so (Fig. 3). Lunule visible in some cases (Fig. 4). Katepisternum mostly with one seta (Fig. 1). One or two notopleural setae. Slender species.

Comments. Micropeza is a large and still poorly known genus. Neotropical species were keyed by Hennig (1936). Several species were subsequently described, thereby making it problematic to determine which species are indeed within this genus.

References. Cresson (1930), Hennig (1936), Aczél (1949).

Brazilian species. Micropeza abbreviata Cresson, 1926 [Brazil, Mato Grosso do Sul, Porto Murtinho, Fazenda Campo Florido*], Micropeza armipennis Bezzi, 1924 [Brazil, Goiás, Campinas (Albuquerque 1966); Mato Grosso do Sul, Serra da Bodoquena, Fazenda Califórnia*; Paraná, Almirante Tamandaré*; Paraná, Castro*; Paraná, Ponta Grossa (Albuquerque 1966); Rio de Janeiro, Angra dos Reis (Albuquerque 1966); Rio de Janeiro, Nova Friburgo (Albuquerque 1966); São Paulo (type-locality); São Paulo, Barueri (Albuquerque 1966); São Paulo, Campos do Jordão (Albuquerque 1966); São Paulo, Osasco (Albuquerque 1966); São Paulo, "Pinheiros" (Albuquerque 1966); São Paulo, "Serra da Cantareira" (Albuquerque 1966)], Micropeza brasiliensis Schiner, 1868 ["Brazil \& Colombia" (type-locality)], Micropeza incisa Wiedemann, 1830 [Brazil (type-locality)], Micropeza pallens Wiedemann, 1830 [Brazil (type-locality)], Micropeza planula Cresson, 1926 [Brazil, Mato Grosso do Sul, Aquidauana*], Micropeza sagittifer Cresson, 1926 [Brazil, Mato Grosso, Chapada dos Guimarães (type-locality); Rondônia, Cacaulândia, Sítio do João Cabeça*; Rondônia, Campo Novo de Rondônia*; Rondônia, Monte Negro*], Micropeza stigmatica Wulp, 1897 [Brazil, Espírito Santo, 

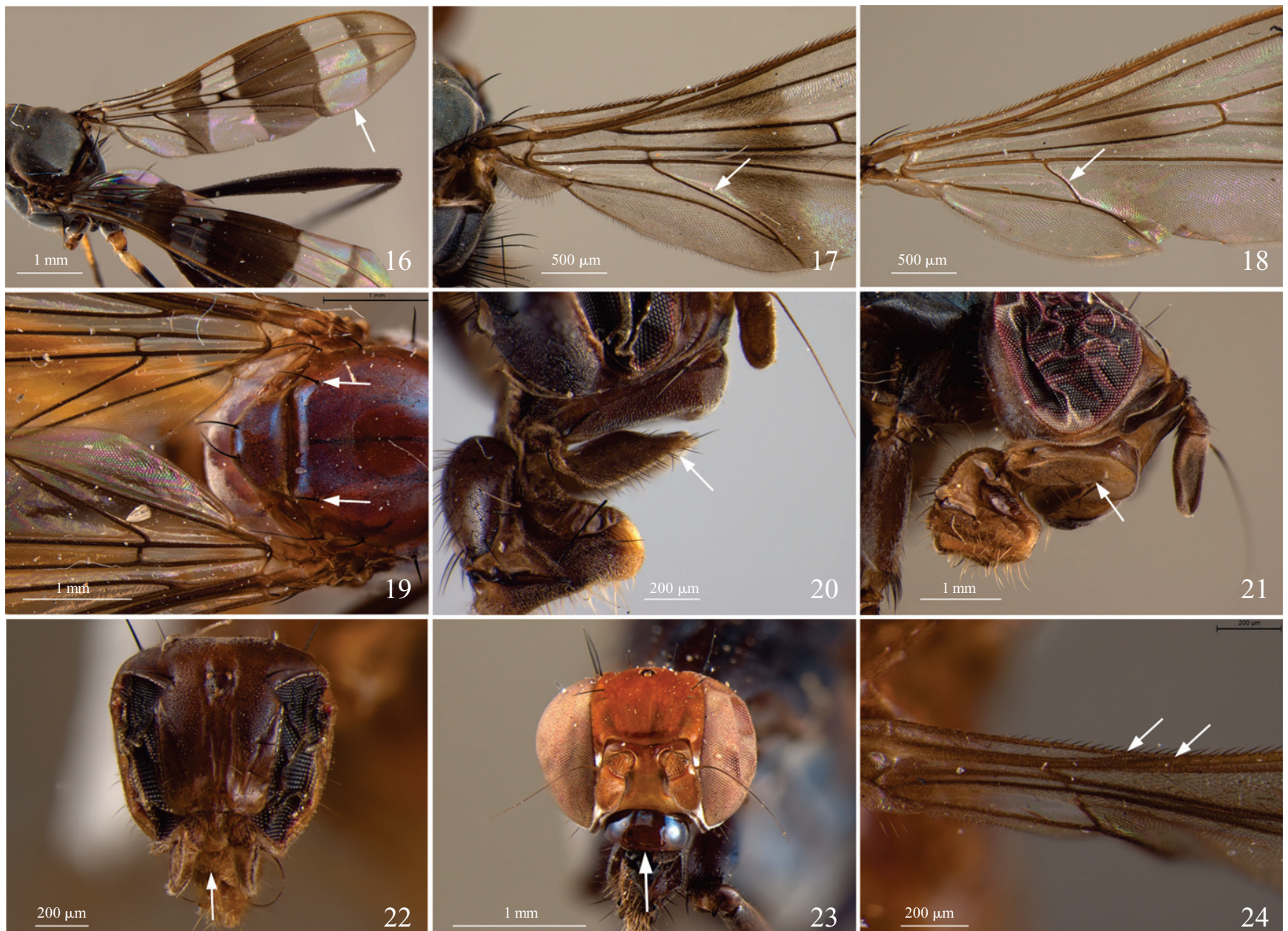

19
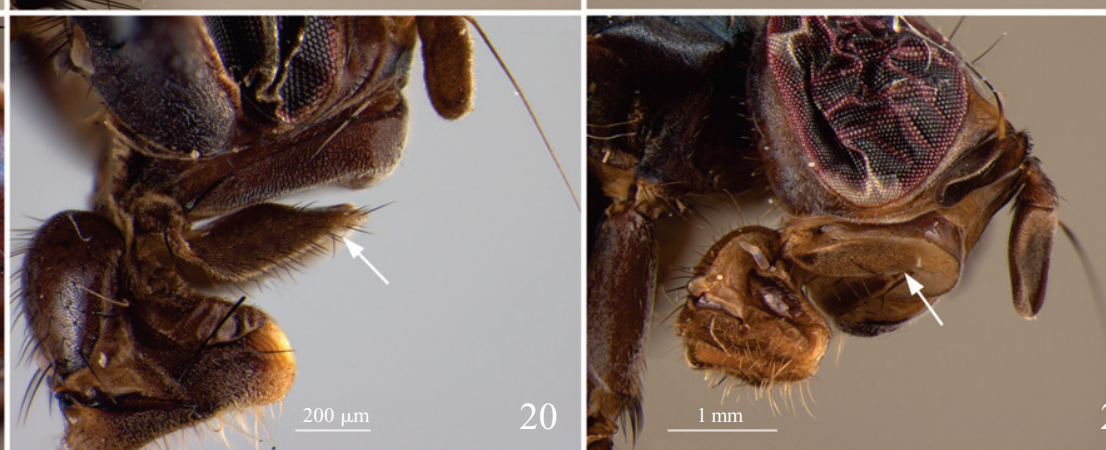

18

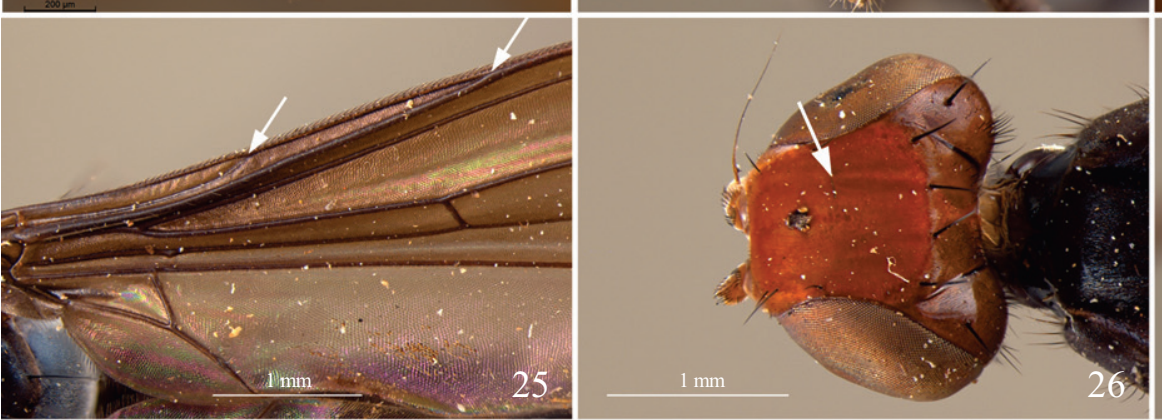

$3 \quad 200 \mu \mathrm{m}$
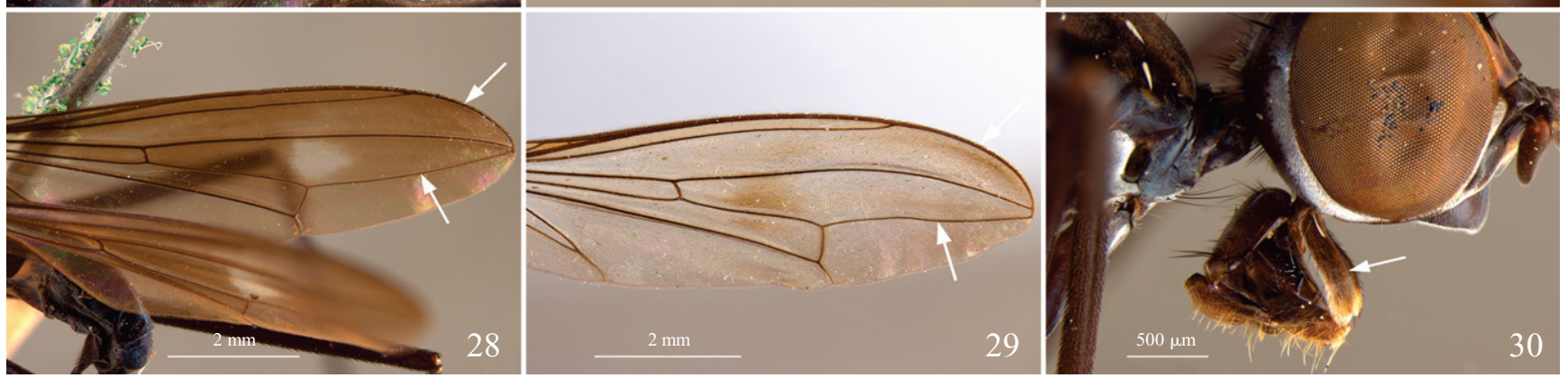

Figs. 16-30. 16. Wing, dorsal view, Plocoscelus brevipennis (arrow: hyaline strip); 17. Wing, dorsal view, Taeniaptera angulata (arrow: CuA 2 vein); 18. Wing, dorsal view, Rainieria paraffinis (arrow: $\mathrm{CuA}_{2}$ vein); 19. Thorax, dorsal view, Ptilosphen fulvus (arrows: dorsocentral setae); 20. Head, lateral view, Taeniaptera angulata (arrow: palpus); 21. Head, ventro-lateral view, Poecilotylus egregius (arrow: palpus); 22. Head, frontal view, Metasphen vigilans (arrow: clypeus); 23. Head, frontal view, Scipopus diversus (arrow: clypeus); 24. Wing, dorsal view, Metasphen vigilans (arrows: Sc and $\mathrm{R}_{1}$ veins); 25. Wing, dorsal view, Scipopus diversus (arrows: $\mathrm{Sc}$ and $\mathrm{R}_{1}$ veins); 26. Head, dorsal view, Scipopus diversus (arrow: limits between epicephala and mesofrons); 27. Head, dorsal view, Cliobata diadema (arrow: limits between epicephala and mesofrons); 28. Wing, dorsal view, Cliobata guttulipennis (arrows: Costa and M veins); 29. Wing, dorsal view, Rainieria paraffinis (arrows: Costa and M veins); 30. Head, lateral view, Cliobata guttulipennis (arrow: palpus). 
Conceição da Barra*; Mato Grosso do Sul, Bodoquena, Fazenda Califórnia*; Mato Grosso do Sul, Porto Murtinho*], Micropeza luteiventris (Czerny, 1932) [Brazil, Pará, Belém (type-locality)], Micropeza maculidorsum (Enderlein, 1922) [Brazil, Rio Grande do Sul, Porto Alegre (type-locality)].

\section{Parasphen Enderlein, 1922}

Parasphen Enderlein, 1922: 226. Type species: Parasphen amazonicus Enderlein, 1922

Diagnose. Arista plumose (Fig. 9). Mesofrons and epicephala not clearly delimited. Dorsocentral setae sometimes absent. Length of Costa between $\mathrm{R}_{2+3}$ and $\mathrm{R}_{4+5}$ less than onehalf as long as $\mathrm{M}$ after dm-cu.

Comments. Parasphen and Ptilosphen represent the micropezids with a plumose arista (Fig. 9) and cell cup long (Fig. 17). Parasphen differs mainly by the limits between mesofrons and epicephala and the wing venation pattern, also indicated by Enderlein (1922), Cresson (1930) and Hennig (1934).

References. Enderlein (1922), Cresson (1930), Hennig (1934)

Brazilian species. Parasphen amazonicus Enderlein, 1922 [Brazil, "Upper Amazon" (type-locality); Amazonas, Manaus, Reserva Ducke*], Parasphen frontalis Cresson, 1930 [Brazil, Pará, Belém*].

\section{Planipeza Marshall, 2008}

(Figs. 32, 33)

Planipeza Marshall, 2008: 189. Type species: Planipeza rafaeli Marshall, 2008.

Diagnosis. Darker species. Anterior portion of thorax elongated, conspicuously surpassing the forecoxa (Fig. 33). Wing mostly dark brown with round hyaline spots (Fig. 32). Male unknown.

Comments. This monotypic genus was described based on one female (Marshall 2008), differing from all other genera mainly because of the elongated anterior portion of thorax (Fig. 33).

References. Marshall (2008).

Brazilian species. Planipeza rafaeli Marshall, 2008 [Brazil, Paraná, São José dos Pinhais (type-locality); São Paulo, Santo André, REBIO Paranapiacaba*].

\section{Plocoscelus Enderlein, 1922}

(Figs. 5, 7, 10, 13, 14, 16)

Plocoscelus Enderlein, 1922: 226. Type species: Calobata arthritica Wiedemann, 1830.

Diagnosis. Paracephala not swollen (Fig. 13), if swollen, head not heart-shaped in dorsal view, the inner-vertical setae are in conspicuous protuberances and the paracephala and epicephala limits are clearly visible (Fig. 14). Wing without hyaline rounded spots in the apical portion (Fig. 16).
Comments. Plocoscelus is an ant-like Neotropical genus easily distinguished by the presence of pectinate arista (Fig. 7) and upturned scutellum (Fig. 5). They differ from Cardiacephala mainly by the lack of heart-shaped head. Also, they may display curious behaviours, as noted by Eberhard (1999).

References. Cresson (1930), Hennig (1935b).

Brazilian species. Plocoscelus brevipennis (Walker, 1853) [Brazil (type-locality); Amapá, Porto Platon*; Amapá, Serra do Navio*; Amazonas, Manaus, Reserva Ducke (Albuquerque 1989); Maranhão, Rosário, Igarapé Paraqueú*; Mato Grosso, Chapada dos Guimarães*; Mato Grosso, Reserva Humboldt*; Minas Gerais, Passos (Albuquerque 1989); Pará, Belém*; Pará, Fordlândia*; Pará, Inhangapi*; Pará, Oriximiná*; Pará, Tucuruí, Vila Brava (Albuquerque 1989); Paraná, Morretes (Albuquerque 1989); Rondônia, Cacaulândia, Sítio do João Cabeça*; Rondônia, Monte Negro, Linha C25 Setor Chacareiro*; Rondônia, Porto Velho, AHE Jirau Rio Madeira*; Roraima, Ilha de Maracá (Albuquerque 1991); Roraima, Pacaraima (Albuquerque 1991); São Paulo, Araçatuba*; São Paulo, Avanhandava, Barra Mansa*], Plocoscelus camptomerus (Bigot, 1886) [Brazil (type-locality)], Plocoscelus cinnameus Hennig, 1935 [Brazil, Acre, Rio Branco*; Amapá, Serra do Navio*; Amazonas, Reserva Ducke*; Rondônia, Porto Velho, AHE Jirau Rio Madeira*], Plocoscelus conifer (Hendel, 1933) [Brazil, Amazonas, Manaus*; Goiás, Corumbá de Goiás*; Mato Grosso, Chapada dos Guimarães*; Mato Grosso, Poconé, Fazenda Rio Clarinho*; Mato Grosso do Sul, Aquidauana, Reserva Ecológica UEMS*; Mato Grosso do Sul, Corumbá*; Mato Grosso do Sul, Porto Murtinho, Fazenda Retiro da Conceição*; Mato Grosso do Sul, Rio Verde, Pousada Quedas D'Água*; Mato Grosso do Sul, Serra da Bodoquena, Fazenda Califórnia*; Minas Gerais, Arceburgo*; Paraná, Umuarama*; Roraima, Ilha de Maracá (Albuquerque 1991); São Paulo, Avanhandava, Barra Mansa*], Plocoscelus modestus Cresson, 1930 [Brazil, Amapá, Serra do Navio*; Pará, Belém, Mocambo*], Plocoscelus niger (Schiner, 1868) [Brazil, Acre, Cruzeiro do Sul, Ilha Florianópolis*; Amazonas, Itacoatiara, Boa Fé*; Amazonas, Manaus, Reserva Ducke*; Amazonas, Urucurituba*; Espírito Santo, Baixo Guandu*; Mato Grosso, Chapada dos Guimarães, Véu da Noiva*; Mato Grosso do Sul, Aquidauana, Reserva Ecológica UEMS*; Mato Grosso do Sul, Serra da Bodoquena, Fazenda Califórnia*; Pará, Tucuruí*; Rio de Janeiro, Rio de Janeiro, Jardim Botânico*; Rondônia, Cacaulândia, Cachoeira Jamari*; Rondônia, Porto Velho, Calama*; Rondônia, Monte Negro, Linha C25 Setor Chacareiro*; Rondônia, Porto Velho, AHE Jirau Rio Madeira*; Roraima, Ilha de Maracá (Albuquerque 1991); São Paulo, Avanhandava*; São Paulo, Castilho*; São Paulo, Severínia*], Plocoscelus nitidus Hennig, 1935 [Brazil (type-locality); Rio de Janeiro, Angra dos Reis, Jussaral*], Plocoscelus podagricus (Rondani, 1848) [Brazil, Pará, Belém (type-locality)], Plocoscelus punctipennis (Macquart, 1843) [Brazil, Rio de Janeiro, Nova Friburgo], Plocoscelus setosus Hennig, 1935 [Brazil, Rondônia, Cacaulândia, Cachoeira Jamari*], Plocoscelus townsendi Cresson, 1930 [Brazil, Amazonas]. 

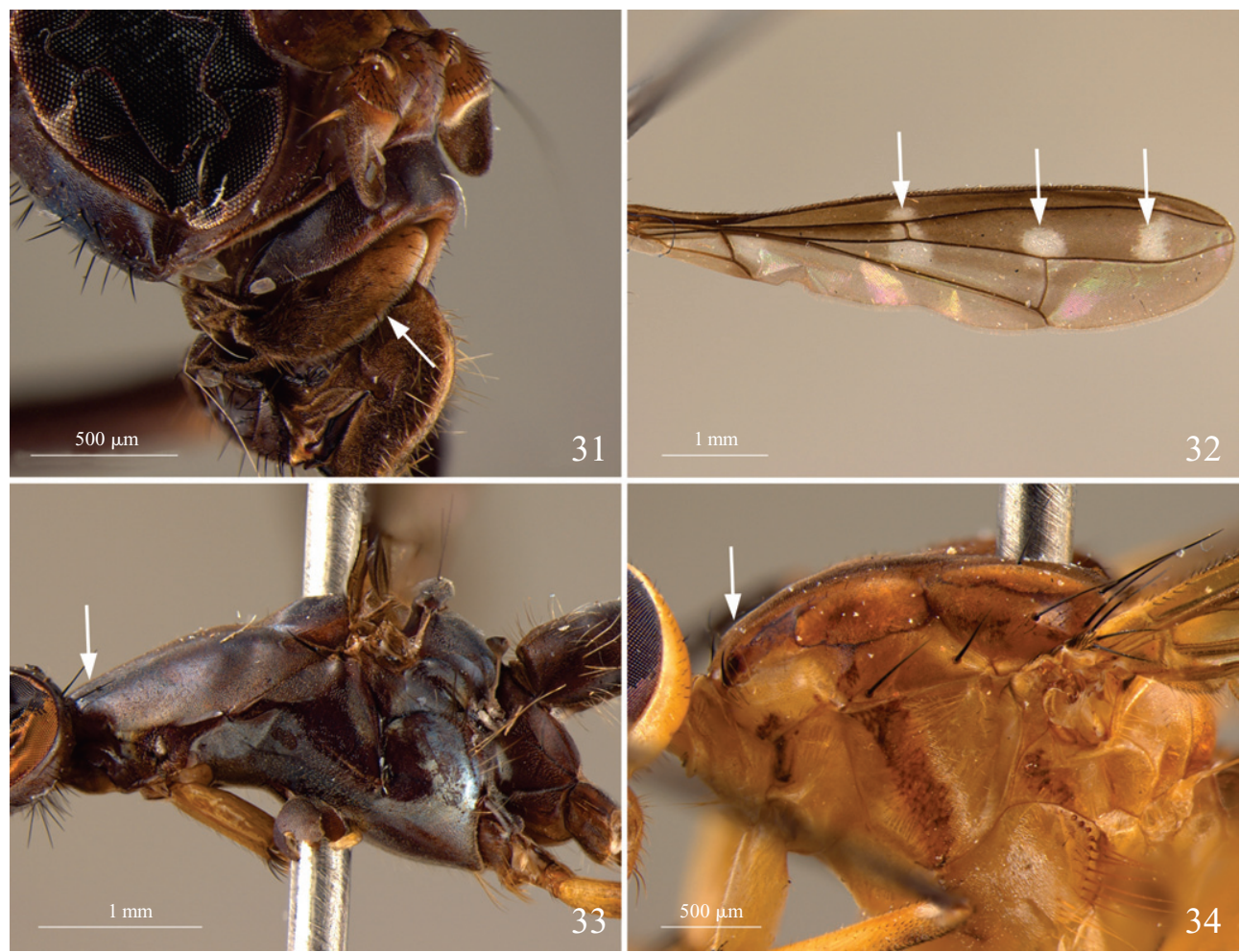

Figs. 31-34. 16. 31. Head, lateral view, Rainieria paraffinis (arrow: palpus); 32. Wing, dorsal view, Planipeza rafaeli (arrows: hyaline spots); 33. Thorax, lateral view, Planipeza rafaeli (arrow: elongated anterior portion of thorax); 34. Thorax, lateral view, Grallipeza nigrinotata (arrow: anterior portion of thorax).

\section{Poecilotylus Hennig, 1934}

(Fig. 21)

Poecilotylus Hennig, 1934: 294. Type species: Calobata varia Wiedemann, 1830

Diagnosis. Arista bare (Fig. 8). Palpus not enlarged, parallel sided with rounded apex (Fig 21). Cell cup long (Fig. 17). Color of wing variable, with darkened crossbands and sometimes with faded or indistinct spots.

Comments. Some species are very similar to others in the genus Taeniaptera. Hennig (1934) supported this genus mostly using the form of the palpus and the faded and indistinct darkened spots in the wing.

References. Hennig (1934).

Brazilian species. Poecilotylus bistrigatus Enderlein, 1922 [Brazil (type-locality)], Poecilotylus egregius Hennig, 1934 [Brazil; Amazonas, Manaus, Reserva Ducke*; Rondônia, Porto Velho, AHE Jirau Rio Madeira*; Roraima, Pacaraima (Albuquerque 1991)], Poecilotylus landrocki (Czerny, 1932) [Brazil, São Paulo, Alto da Serra (type-locality)], Poecilotylus luridilabris Enderlein, 1922 [Brazil, "Upper Amazon” (typelocality); Amapá, Serra do Navio*; Amazonas, Manaus, Reserva Ducke*; Pará, Belém*], Poecilotylus obliquus (Fabricius, 1805) [Brazil], Poecilotylus paraguayensis (Enderlein, 1922) [Brazil, Paraná, Jussara*; Rondônia, Cacaulândia, Sítio do João Cabeça*; Rondônia, Cacaulândia, Cachoeira Jamari*; Rondônia, Monte Negro, Linha C25 Setor
Chacareiro*; Rondônia, Porto Velho, Área Abunã A11P1*; Rondônia, Porto Velho, AHE Jirau Rio Madeira*;], Poecilotylus similis Hennig, 1934 [Brazil,; Espírito Santo, Santa Teresa*; Santa Catarina, Nova Teutônia, Seara*; São Paulo, Alto da Serra (type-locality)], Poecilotylus testaceus (Fabricius, 1805) [Brazil, Amapá, Serra do Navio, Rio Amapari*; Amazonas, Manaus, Reserva Ducke*; Pará, Belém, Fazenda Velha*; Paraná, Altamira do Paraná, Estrada Água da Bota*; Paraná, Morretes*; Rondônia, Cacaulândia, Sítio do João Cabeça*; Rondônia, Porto Velho, AHE Jirau Rio Madeira*], Poecilotylus trifasciatus (Wiedemann, 1830) [Brazil (type-locality)], Poecilotylus varius (Wiedemann, 1830) [Brazil (type-locality); Mato Grosso, Chapada dos Guimarães*; Rondônia, "Rio Madeira"*].

\section{Ptilosphen Enderlein, 1922}

(Fig. 19)

Ptilosphen Enderlein, 1922: 222. Type species: Calobata insignis Wiedemann, 1830.

Diagnosis. Arista plumose (Fig. 9). Mesofrons and epicephala clearly delimited, in most cases with a blackened velvety spot. Dorsocentral setae present (Fig. 19). Length of Costa between $\mathrm{R}_{2+3}$ and $\mathrm{R}_{4+5}$ more than one half as long as $\mathrm{M}$ after dm-cu.

Comments. This Neotropical genus is recognized by the following combination of characters, including cell cup long 
(Fig. 17) and plumose arista (Fig. 9). This genus shares these characters with Parasphen, but only Ptilosphen has a clearly delimited mesofrons and epicephala.

References. Cresson (1930), Hennig (1934).

Brazilian species. Ptilosphen comis Cresson, 1930 [Brazil, Pará, Belém; Pernambuco, Recife; Roraima, Ilha de Maracá (Albuquerque 1991); Roraima, Pacaraima (Albuquerque 1991)], Ptilosphen dubius Hennig, 1934 [Brazil, Amazonas, Manaus, AM010 km 31 Embrapa*; Amazonas, Manaus*; Roraima, Ilha de Maracá (Albuquerque 1991); Roraima, Pacaraima (Albuquerque 1991)], Ptilosphen enderleini Cresson, 1930 [Brazil, Rondônia, Porto Velho, $A H E$ Jirau Rio Madeira*], Ptilosphen fulvus (Walker, 1849) [Brazil, Amazonas, Manaus, Reserva Ducke*; Goiás, Corumbá de Goías*; Goiás, Goiânia*; Maranhão, Santa Helena, Alto Tui*; Mato Grosso, Aripuanã, Estrada Serraria*; Pará (typelocality); Rondônia, Ouro Preto do Oeste*], Ptilosphen insignis (Wiedemann, 1830) [Brasil, (type-locality); Acre, Rio Branco*; Amazonas, Manaus, Reserva Ducke*; Amazonas, "Tapuruquara, Rio Negro"*; Pará, Belém, Utinga*; Rondônia, Cacaulândia, Sítio do João Cabeça*; Roraima, Ilha de Maracá (Albuquerque 1991)], Ptilosphen rufifrons Enderlein, 1922 [Brazil, Rondônia, Monte Negro, Linha C25 Setor Chacareiro*], Ptilosphen tetrastigma (Schiner, 1868) [Brazil].

\section{Rainieria Rondani, 1843}

(Figs. 18, 29, 31)

Rainieria Rondani, 1843: 40. Type species: Calobata calceata Fallen, 1820.

Diagnosis. Palpus very enlarged (Fig. 31). Length of Costa between $\mathrm{R}_{2+3}$ and $\mathrm{R}_{4+5}$ more than one half as long as $\mathrm{M}$ after dm-cu (Fig. 29). Peduncle of $\mathrm{CuP}$ vein not visible in cup cell. Hyaline wing, in most cases with darkened crossbands or spots, but never totally infuscated.

Comments. This genus was characterized by Cresson (1930) and Hennig (1935b) by its broad palpus (Fig. 31) and length of Costa between $\mathrm{R}_{2+3}, \mathrm{R}_{4+5}$ and $\mathrm{M}$, after dm-cu (Fig. 29). Also, Hennig (1935b) created the subgenus Rainierella to include the species with only one frontal setae.

References. Cresson (1930), Hennig (1935b).

Brazilian species. Rainieria alternata Cresson, 1926 [Brazil]; Rainieria paraffinis Hennig, 1935 [Brazil, Mato Grosso, Chapada dos Guimarães*; Mato Grosso do Sul, Aquidauana, Reserva Ecológica UEMS*; Mato Grosso do Sul, Porto Murtinho, Fazenda Retiro da Conceição*; Pernambuco, "Fazenda Caruaru"*; Rondônia, Cacaulândia, Sitio do João Cabeça*; Rondônia, Porto Velho, AHE Jirau Rio Madeira*; Rondônia, Porto Velho, Área Caiçara C3P4*; Roraima, Ilha de Maracá (Albuquerque 1991); Roraima, Pacaraima (Albuquerque 1991)], Rainieria soccata (Enderlein, 1922) [Brazil, "Upper Amazon"(type-locality)], Rainieria uniformis Hennig, 1935 [Brazil, Santa Catarina, Blumenau (type-locality)].

\section{Scipopus Enderlein, 1922}

(Figs. 23, 25, 26)

Scipopus Enderlein, 1922: 208. Type species: Calobata erythrocephala Fabricius, 1805.

Diagnosis. Larger and always darker species, sometimes with shining metallic blue. Divisions between mesofrons and epicephala visible (Fig. 26). Head usually orange. Wing totally infuscated, rarely with hyaline spots.

Comments. Scipopus is a Neotropical genus, easily distinguished by darker color and robust body (over $15 \mathrm{~mm}$ in length). Unlike Cliobata, Scipopus has a strong orange color in head, and no divisions between mesofrons and epicephala (Fig. 26).

References. Cresson (1930), Hennig (1934).

Brazilian species. Scipopus alvarengai Albuquerque, 1972 [Brazil, Pará, Jacareacanga (type-locality)], Scipopus belzebul (Schiner, 1868) [Brazil, Amazonas, Itacoatiara (Albuquerque 1972); Amazonas, São Gabriel da Cachoeira (Albuquerque 1972); Amazonas, Tabatinga (Albuquerque 1972); Amazonas, Manaus, Reserva Ducke (Albuquerque 1972); Amapá, Porto Platon*; Maranhão, Imperatriz*; Pará, Belém, Utinga (Albuquerque 1972); Pará, Capanema (Albuquerque 1972); Pará, "Estrada de Ferro Belém-Bragança” (Albuquerque 1972); Pará, Jacareacangá (Albuquerque 1972); Pará, Oriximiná, Boca do Cuminá-Miri*; Pará, São Luís, Rio Tapajós*; Rondônia, Cacaulândia, Sítio do João Cabeça*; Rondônia, Porto Velho, Mutum Paraná*], Scipopus calocephala (Bigot, 1886) [Brazil], Scipopus cartaboensis Cresson, 1926 [Brazil, Espírito Santo, Linhares*; Mato Grosso do Sul, Serra da Bodoquena, Fazenda Califórnia*; Rondônia, Porto Velho, AHE Jirau Rio Madeira*; Rondônia, Porto Velho, Mutum Paraná*], Scipopus diversus (Schiner, 1868) [Brazil, Amazonas, Manaus, "BR174 (antiga BR17" (Albuquerque 1971); Amazonas, Manaus, Reserva Ducke (Albuquerque 1971); Amazonas, São Gabriel da Cachoeira (Albuquerque 1971); Amazonas, Tabatinga (Albuquerque 1971); Amapá, Porto Platon*; Espírito Santo, Colatina*; Espírito Santo, Conceição da Barra (Albuquerque 1971); Espírito Santo, Baixo Guandu*; Espírito Santo, Linhares*; Espírito Santo, Ribeiro de Figueiredo (Albuquerque 1971); Goiás, Goiânia, Campinas (Albuquerque 1971); Mato Grosso, Chapada dos Guimarães*; Mato Grosso, Utiariti*; Mato Grosso do Sul, Iguatemi*; Mato Grosso do Sul, Serra da Bodoquena, Fazenda Califórnia*; Maranhão, Rosário, Igarapé Paraqueú*; Minas Gerais (Albuquerque 1971); Minas Gerais, Arceburgo*; Minas Gerais, Buritis, Ribeirão Confins; Pará, Cachimbo*; Pará, Belém (Albuquerque 1971); Pará, Taperinha; Paraná, Foz do Iguaçu*; Paraná, Morretes*; Paraná, Terra Boa*; Rondônia, Porto Velho*; Rondônia, Porto Velho, Mutum Paraná*; Rondônia, Vilhena*; Roraima, Ilha de Maracá (Albuquerque 1991); São Paulo, Avanhandava, Barra Mansa*; São Paulo, Ribeirão Preto*], Scipopus erythrocephalus (Fabricius, 1805) [Brazil, Amapá, Porto Platon*; Amazonas, Manaus, “BR 17” (Albuquerque 1971); 
Amazonas, Manaus, Reserva Ducke (Albuquerque 1971); Amazonas, Tabatinga (Albuquerque 1971); Espírito Santo, Linhares*; Pará, Belém*; Piauí; Rondônia, Monte Negro, Linha C25 Setor Chacareiro*; Rondônia, Porto Velho, AHE Jirau Rio Madeira*; Rondônia, Vilhena*], Scipopus frit Cresson, 1926 [Brazil, Rondônia, Cacaulândia*], Scipopus heteropus (Frey, 1927) [Brazil], Scipopus limbativertex Enderlein, 1922 [Brazil, Mato Grosso], Scipopus souzalopesi Albuquerque, 1972 [Brazil, Acre (type-locality)], Scipopus striatithorax Hennig, 1934 [Brazil, Espírito Santo, Conceição da Barra*; Espírito Santo, Linhares*; Minas Gerais (typelocality)].

\section{Taeniaptera Macquart, 1835}

(Figs. 2, 6, 8, 17, 20)

Taeniaptera Macquart, 1835: 491. Type species: Taeniaptera trivittata Macquart, 1835

Diagnosis. Arista bare (Fig. 8). Palpus enlarged basally, tapering to apex (Fig. 20). Cell cup long (Fig. 17). Color of wing variable, but mostly with darkened spots or crossbands.

Comments. All species of Taeniaptera have cell cup long (Fig. 17) and bare arista (Fig. 8), differing from Poecilotylus by the enlarged basal portion of the palpus (Fig. 20). Most species have a strongly darkened discal wing band.

References. Cresson (1930), Hennig (1934).

Brazilian species. Taeniaptera albibasis Enderlein, 1922 [Brazil, Amazonas, AM1 km 104 (Albuquerque 1981); Amazonas, "BR174 (antiga BR17)" (Albuquerque 1981); Amazonas, "Estrada Aleixo Pomar" (Albuquerque 1981); Amazonas, Manaus, Reserva Ducke (Albuquerque, 1981); Espírito Santo, Baixo Guandu*; Mato Grosso, Chapada dos Guimarães*; Mato Grosso, Aripuanã, Reserva Humboldt*; Mato Grosso, Utiariti, Rio Papagaio*; Mato Grosso do Sul, Corumbá*; Minas Gerais, Coronel Fabriciano*; Pará, BelémBragança km 100 (Albuquerque 1981); Pará, Belém-Brasília km 90 (Albuquerque 1981); Pará, Belém, Utinga (Albuquerque 1981); Rondônia, Porto Velho, Área Mutum*; Rio de Janeiro, Grajaú (Albuquerque 1981); Rio de Janeiro, "Palmeiras" (Albuquerque 1981); Rio de Janeiro, Rio de Janeiro, Represa Rio Grande*], Taeniaptera albitarsis Enderlein, 1922 [Brazil, "Upper Amazon" (type-locality); Amazonas, Manaus, Reserva Ducke (Albuquerque 1980b); Amazonas, AM1 km 16 (Albuquerque 1980b); Amazonas, Manaus, Campus do INPA (Albuquerque 1980b); Amazonas, Manaus, "Reserva Biológica Campina, BR174" (Albuquerque 1980b); Amapá, Serra do Navio*; Espírito Santo, Baixo Guandu*; Espírito Santo, Santa Teresa*; Mato Grosso do Sul, Serra da Bodoquena, Fazenda Califórnia*; Mato Grosso do Sul, Três Lagoas, Fazenda Floresta*; Minas Gerais, Arceburgo*; Pará, Belém (Albuquerque 1980b); Pará, "Fazenda Velha" (Albuquerque 1980b); Pará, Jambuaçú*; Pará, Bragança, Santa Maria (Albuquerque 1980b); Pará, Belém, Utinga (Albuquerque 1980b); Pernambuco, "Fazenda Caruaru"*; Rio de Janeiro, Angra dos Reis*; Rio de Janeiro,
Rio de Janeiro, Grajaú (Albuquerque 1980b); Rio de Janeiro, Rio de Janeiro, Represa Rio Grande*; Santa Catarina, Blumenau*; Santa Catarina, Joinville*; São Paulo, Boracéia (Albuquerque 1980b); São Paulo, Juquiá*], Taeniaptera aliceae Albuquerque, 1980 [Brazil, Amazonas, Manaus (typelocality); Paraná, Morretes*; Roraima, Ilha de Maracá (Albuquerque 1991)], Taeniaptera angulata (Loew, 1866) [Brazil, Amazonas, AM01 km 182 (Albuquerque 1981); Amazonas, "BR174 (antiga BR17)" (Albuquerque 1981); Amazonas, Manaus, Campus do INPA (Albuquerque 1981); Amazonas, Manaus, Estrada Aleixo-Mauá (Albuquerque 1981); Amazonas, Manaus, Reserva Ducke (Albuquerque 1981); Amazonas, Tabatinga (Albuquerque 1981); Goiás, Goiânia, Campinas*; Mato Grosso, Aripuanã, Reserva Humboldt (Albuquerque 1981); Mato Grosso, Cáceres*; Mato Grosso, Chapada dos Guimarães*; Mato Grosso do Sul, Aquidauana, Reserva Ecológica UEMS*; Mato Grosso do Sul, Bodoquena*; Mato Grosso do Sul, Corguinho, Taboco, Reserva Quinta do Sol*; Mato Grosso do Sul, Rio Verde, Pousada Quedas D’Água*; Minas Gerais, Lassance (Albuquerque 1981); Minas Gerais, Além Paraíba, Porto Novo do Cunha*; Pará, Belém (Albuquerque 1981); Pará, Marituba*; Pará, Tucuruí, Paraquequara*; Rio de Janeiro, Rio de Janeiro, Manguinhos (Albuquerque 1981); Rio de Janeiro, Nova Iguaçu (Albuquerque 1981); Rondônia, Cacaulândia*; Rondônia, Campo Novo de Rondônia, Fazenda Amorim*; Rondônia, Monte Negro, Linha C25 Setor Chacareiro*; Rondônia, Porto Velho, AHE Jirau Rio Madeira*; Roraima, Ilha de Maracá (Albuquerque 1991); Roraima, Pacaraima (Albuquerque 1991); São Paulo, Juquiá*; São Paulo, Peruíbe*], Taeniaptera annulata (Fabricius, 1805) [Brazil, Amazonas, Estrada AM1 km 155 (Albuquerque 1981); Amazonas, "BR174 (antiga BR17)" (Albuquerque, 1981); Amazonas, BR17 km 63 (antiga BR17) (Albuquerque 1981); Amazonas, Manaus, Colônia Santo Antonio (Albuquerque 1981); Amazonas, Manaus, Estrada Aleixo-Mauá (Albuquerque 1981); Amazonas, Manaus, Reserva Ducke (Albuquerque 1981); Amazonas, Tabatinga (Albuquerque, 1981); Bahia, Salvador (Albuquerque 1981); Distrito Federal, Brasília (Albuquerque 1981); Espírito Santo, Linhares*; Espírito Santo, Santa Teresa*; Goiás, Corumbá de Goiás*; Goiás, Goiânia*; Mato Grosso do Sul, Serra da Bodoquena, Fazenda Califórnia*; Pará, Belém (Albuquerque 1981); Pará, "Estrada Belém-Brasília" (Albuquerque 1981); Pará, Belém, Utinga (Albuquerque 1981); Paraná, Castro*; Paraná, Curitiba*; Paraná, Guaratuba*; Paraná, Morretes*; Paraná, Rio Negro*; Paraná Umuarama*; Rio de Janeiro, Itatiaia, Campo Belo*; Rio de Janeiro, Rio de Janeiro, Represa Rio Grande*; Rio de Janeiro, "Palmeiras" (Albuquerque 1981); Rio de Janeiro, Resende*; Rio de Janeiro, Teresópolis*; Rio Grande do Sul, Barão de Cotegipe*; São Paulo, Araçatuba*; São Paulo, São Paulo*], Taeniaptera diversicolor (Macquart, 1855) [Brazil, Amazonas (type-locality)], Taeniaptera inornata Hennig, 1934 [Brazil, Amazonas, Coari, "Rio Solimões" (type-locality)], Taeniaptera inpai Albuquerque, 1980 [Brazil, Amazonas, São Gabriel da Cachoeira (type- 
locality)], Taeniaptera lasciva (Fabricius, 1798) [Brazil, Amapá, Serra do Navio*; Bahia, Salvador*; Amazonas, Manaus, Reserva Ducke (Albuquerque 1980a); Amazonas, Manaus, Estrada Aleixo Lajes (Albuquerque 1980a); Amazonas, "AM1 km 104" (Albuquerque 1980a); Amazonas, Manaus, Colonia Santo Antônio (Albuquerque 1980a); Amazonas, Manaus, Campus do INPA (Albuquerque 1980a); Amazonas, "BR174 (antiga BR17)" (Albuquerque 1980a); Amazonas, Tabatinga (Albuquerque 1980a); Distrito Federal, Brasília (Albuquerque 1980a); Mato Grosso, Aripuanã (Albuquerque 1980a); Mato Grosso, Chapada dos Guimarães, Trilha da Pedra*; Mato Grosso, Aripuanã, Reserva Humboldt*; Mato Grosso do Sul, Corumbá, Passo do Lontra $B E P^{*}$; Mato Grosso do Sul, Campo Grande*; Minas Gerais, Cambuquira*; Minas Gerais, Diamantina*; Minas Gerais, Governador Valadares*; Minas Gerais, Passos*; Pará, Breves, Ilha de Marajó*; Pará, "Belém-Brasília" (Albuquerque 1980a); Pará, Salinas (Albuquerque 1980a); Paraná, Curitiba*; Paraná, "Marumbi”*; Paraná, Matinhos*; Paraná, Terra Boa*; Rio de Janeiro, Petrópolis*; Rio Grande do Sul, Barão de Cotegipe*; Rio de Janeiro, Vassouras (Albuquerque 1980a); Rio Grande do Sul, Porto Alegre (Albuquerque 1980a); Rondônia, Monte Negro, estação da USP*; Roraima, Caracarai*; Roraima, Ilha de Maracá (Albuquerque 1991); Roraima, Pacaraima (Albuquerque 1991); São Paulo, Araçatuba*; São Paulo, Barueri (Albuquerque 1980a); São Paulo, Embú*], Taeniaptera longifurca Hendel, 1933 [Brazil, Rio de Janeiro, Petrópolis (type-locality)], Taeniaptera nigriceps Hennig, 1934 [Brazil (type-locality)], Taeniaptera nigritarsis (Macquart, 1843) [Brazil (type-locality)]; Taeniaptera parens Cresson, 1926 [Brazil], Taeniaptera postannulus Enderlein, 1922 [Brazil, Espírito Santo (typelocality); Rondônia, Cacaulândia, Sítio do João Cabeça*; Rondônia, Monte Negro, Linha C25 Setor Chacareiro*], Taeniaptera rufifacies (Macquart, 1851) [Brazil, Amapá, Serra do Navio*; Pará, Fordlândia, Alto do Tapajós*; Rondônia, Porto Velho, AHE Jirau Rio Madeira*], Taeniaptera simillima (Hendel, 1922) [Brazil], Taeniaptera tarsata (Wiedemann, 1830) ["Brazil and Suriname" (type-locality); Amapá, Serra do Navio*; Amazonas, "Lago de Tefé" (Albuquerque 1980b); Espírito Santo, Baixo Guandu*; Espírito Santo, Linhares*; Goiás, Corumbá de Goiás, Fazenda Monjolinho*; Maranhão, Rosário, Igarapé Paraqueú*; Mato Grosso, Miranda, Salobra*; Pará, Belém, "IAN" (Albuquerque 1980b); Pará, "Ilha das Onças" (Albuquerque 1980b); Pará, Belém, "Museu Goeldi” (Albuquerque 1980b); Pará, Bragança, Santa Maria (Albuquerque 1980b); Paraná, Paranaguá, Alexandra*; Paraná, Morretes*; Paraná, Pinhão*; Rio de Janeiro, Angra dos Reis, Japuiba (Albuquerque 1980b); Rio de Janeiro, "Palmeiras" (Albuquerque 1980b); Rio de Janeiro, Rio de Janeiro, Represa Rio Grande*; Rio de Janeiro, "Tinguá" (Albuquerque 1980b); Santa Cararina, Joinville*; São Paulo, Caraguatatuba*], Taeniaptera teresacristinae Albuquerque, 1981 [Brazil, Amazonas, Manaus (type-locality); Roraima, Ilha de Maracá (Albuquerque 1991); Roraima, Pacaraima (Albuquerque 1991)],
Taeniaptera thiemei (Enderlein, 1922) [Brazil, Mato Grosso, Chapada dos Guimarães, Véu da Noiva*; Rondônia, Cacaulândia, Trilha Cachoeira Jamari*; Rondônia, Monte Negro, Linha C25 Setor Chacareiro*; Rondônia, Porto Velho, AHE Jirau Rio Madeira*], Taeniaptera tibialis (Macquart, 1843) [Brazil, Amazonas, Estrada do Aleixo AM010 km 104 (Albuquerque 1980b); Amazonas, Manaus, Colônia Santo Antônio (Albuquerque 1980b); Amazonas, Manaus, Reserva Ducke (Albuquerque 1980b); Bahia; Espirito Santo, Linhares*; Mato Grosso, Chapada dos Guimarães, Trilha da Pedra*; Mato Grosso do Sul, Aquidauana, Reserva Ecológica UEMS*; Mato Grosso do Sul, Miranda, Fazenda Guaicurus*; Pará, Capanema*; Pará, Oriximiná, Rio Trombetas*; Pará, Santa Maria (Albuquerque 1980b); Pará, Tucuruí*; Paraná, Morretes*; Rio de Janeiro, Manguinhos (Albuquerque 1980b); Rondônia, Cacaulândia, Sítio do João Cabeça*; Rondônia, Monte Negro, Linha C25 Setor Chacareiro*; Rondônia, Porto Velho, AHE Jirau Rio Madeira*; Roraima, Caracaraí*; Roraima, Ilha de Maracá (Albuquerque 1991); Roraima, Pacaraima (Albuquerque 1991); Santa Catarina, Nova Teutônia, Seara*; São Paulo, Registro*], Taeniaptera vittipennis (Coquillett, 1902) [Brazil], Taeniaptera vulgata Hennig, 1934 [Brazil, Pará (type-locality); Roraima, Ilha de Maracá (Albuquerque 1991); Roraima, Pacaraima (Albuquerque 1991)], Taeniaptera vulpes Cresson, 1926 [Brazil, Mato Grosso do Sul, Porto Murtinho, Fazenda Retiro Conceição, Trilha da Espinhadeira*; Paraná, Morretes*].

\section{ACKNOWLEDGEMENTS}

For access to collections and the loans of specimens we thank Carlos J. E. Lamas (MZUSP), Márcio Luiz de Oliveira and José Albertino Rafael (INPA). We are grateful to the TAXon Line project, for making possible the image capturing and the Conselho Nacional de Desenvolvimento Cientifico e Tecnológico $(\mathrm{CNPq})$ for the fellowships (GBF, process number 133126/2012-9; CJBC, process number 304713/2011-2). Thanks also toCNPq(Grant 563256/2010-9) and Fundação de Amparo a Pesquisa do Estado de São Paulo (FAPESP) (Grant 2010/52314-0) for the support to the SISBIOTA Brasil Program. James J. Roper reviewed the English.

\section{REFERENCES}

Aczél, M.L. 1949. Notes on Tylidae, II. Argentine species of the subfamily Tylinae in the Entomological Collection of the Miguel Lillo foundation. Acta Zoologica Lilloana 8: 219-282.

Aczél, M.L. 1951. Morfologia externa y división sistematica de las Tanypezidiformes con sinopsis de las especies argentinas de Tylidae (Micropezidae) y Neriidae (Dipt.). Acta Zoologica Lilloana 11: 483 589.

Albuquerque, L. P. 1966. Observações sobre Protylos armipennis (Bezzi, 1924) (Diptera - Micropezidae). Revista Brasileira de Biologia 26: 39-45.

Albuquerque, L. P. 1971. Contribuição ao conhecimento das espécies do gênero Scipopus Enderlein, 1922 (Diptera - Micropezidae). Acta Amazonica 1: 89-96.

Albuquerque, L.P. 1972. Sobre uma espécie do Gênero Scipopus Enderlein, 1922 (Diptera - Micropezidae). Acta amazonica 2: 89-94. 
Albuquerque, L. P. 1980a. Estudos dos micropezídeos da Bacia Amazônica. 1 - Contribuição à sistemática de Micropezidae e estudo do gênero Taeniaptera Macquart, 1835 (Diptera - Acalyptratae). Acta Amazonica 10: 659-670.

Albuquerque, L. P. 1980b. Estudo dos micropezídeos da Bacia Amazônica. 2 - Redescrição de três espécies e conhecimento de duas espécies novas para a ciência de Taeniaptera Macquart, 1835 (Diptera - Micropezidae). Acta Amazonica 10: 863-881.

Albuquerque, L. P. 1981. Estudo dos micropezídeos da Bacia Amazônica 3 - Conhecimento de uma nova espécie e redescrição de três espécies de Taeniaptera Macquart, 1835 (Diptera-Micropezidae). Acta Amazonica 11: $821-838$.

Albuquerque, L. P. 1989. Cardiacephalina subtr. n. de Taeniapterini (Diptera, Micropezidae, Taeniapterinae). Memórias do Instituto Oswaldo Cruz 84: 19-26.

Albuquerque, L. P. 1991. Taeniapterinae da Ilha de Maracá e de uma localidade de Pacaraima, Roraima Brasil. Acta Amazonica 21: 3-13.

Amorim, D.S., Silva, V.C. \& Balbi, M.I.P.A. 2002. Estado do Conhecimento dos Diptera Neotropicais, p. 29-36. In: Costa, C.,Vanin, S.A., Lobo, J.M. \& Melic, A. (Orgs.). PRIBES. Zaragoza, Sociedad Entomológica Aragoneza, $329 \mathrm{p}$

Cresson, E.T., Jr. 1930. Notes on and descriptions of some neotropical Neriidae and Micropezidae (Diptera). Transactions of the American Entomological Society 56: 307-362

Eberhard, W.G. 1999. Mortal mimicry in the fly, Plocoscelus sp. (Diptera, Micropezidae). Biotropica 31: 535.

Enderlein, G. 1922. Klassifikation der Micropeziden. Archivfür Naturgeschichte A 88: 140-229.

Enderlein, G. 1923. Neue Beiträge zur Kenntnis der Micropeziden (Dipt.). Deutsche Entomologische Zeitschrift 5: 540-543.

Fischer, C.R. 1932. Contribuição para o conhecimento da metamorfose e posição sistemática da família Tylidae (Micropezidae, Dipt.). Revista de Entomologia 2: 16-24.

Frey, R. 1927. Zur Systematik der Diptera Haplostomata. III. Fam. Micropezidae. Notulae Entomologicae Helsingfors 7: 65-76.

Hennig, W. 1934. Revision der Tyliden (Dipt., Acalypt.). Stettiner Entomologische Zeitung 95: 65-108, 294-330.

Hennig, W. 1935a. Revision der Tyliden (Dipt., Acalypt.). II. Teil: die ausseramerikanischen Taeniapterinae, die Trepidariinae und Tylinae. Allgemeines über die Tyliden. Zugleich ein Beitrag zu den Ergebnissen der Sunda-expedition Rensch, 1927 [part]. Konowia 14: 68-92.

Hennig, W. 1935b. Revision der Tyliden (Dipt., Acalypt.). I. Teil: Die Taeniapterinae Amerikas. Stettiner Entomologische Zeitung 96: 27-67.

Hennig, W. 1936. Revision der Tyliden (Dipt., Acalypt.). II. Teil: die ausseramerikanischen Taeniapterinae, die Trepidariinae und Tylinae. Allgemeines über die Tyliden. Zugleich ein Beitrag zu den Ergebnissen der Sunda-expedition Rensch, 1927. Konowia 15: 129-144, 201-239.
Hennig, 1937a. Uebersicht über die Arten der Neriiden und über die Zoogeographie dieser Acalyptraten-Gruppe. Stettiner Entomologische Zeitung 98: 240-280.

Hennig, W. 1937b. Nachtrage zur "Revision der Tyliden.”. Stettiner Entomologische Zeitung 98: 46-50.

Hennig, W. 1937c. Tylidae (Micropezidae, Diptera) aus der Sammlung des British Museum of Natural History. Nachtrage zur " Revision der Tyliden. - II.” Annals and Magazine of Natural History Series (10) 19: $521-523$.

Macquart, J.P. 1835. Histoire Naturele des Insectes.Diptères. Tome deuxieme, Paris, Librairie Encyclopédique de Roret, 703 p.

Macquart, J.P. 1843. Diptères exotiques nouveaux ou peu connus. Mémoires de la Société des Sciences de l'Agriculture et des Arts de Lille 2:162-460.

Marshall, S.A. 2004. A review of the genus Metasphen Frey including Globomyia Hennig syn. nov. (Diptera, Micropezidae). Studia Dipterologica 11: 529-536.

Marshall, S.A. 2008. A monotypic new genus of micropezid fly from Brazil (Diptera, Micropezidae, Taeniapternae). Studia Dipterologica 15: 189-192.

Marshall, S.A. 2010. Micropezidae (stilt-legged flies). In: Brown, B.V., Borkent, A., Cumming, J.M., Wood, D.M., Woodley, N.E. \& Zumbado, M. (Eds.). Manual of Central American Diptera Vol. 2. Ottawa, NRC Research Press, 728 p.

Marshall, S.A. 2012. Flies: the Natural History and Diversity of Diptera. Ontario, Firefly Books, 616 p.

McAlpine, J.F. 1981. Morphology and Terminology - Adults, p. 9-63. In: McAlpine, J.F., Peterson, B.V., Shewell, G.E., Teskey, H.J., Vockeroth, J.R. \& Wood, D.M. (Eds.). Manual of Nearctic Diptera. Monograph 27, vol. 1, Ottawa, Research Branch, Agriculture Canada, 674p.

Meigen, J. W. 1803. Versuch einer neuen Ganttungseintheilung der europäischen zweiflügeligen Insekten. Magazin für Insektenkunde 2: 259-281.

Rondani, C. 1843. Quattro specie di insetti proposti come tipi di generi nuovi. Memoria sesta per servir alla ditterologia italiana. (Accad. Sci. Inst. Bologna). Nuovi Annali delle Scienze Naturali 10: 32-46.

Rondani, C. 1850. Observazioni sopra alquante specie di Esapodi Ditteri Del Museo Torinese. Nuovi Annali delle Scienze Naturali 2:165197.

Steyskal, G.C. 1964. Larvae of Micropezidae (Diptera) including two species that bore in ginger roots. Annals of the Entomological Society of America 57: 292-296.

Steyskal, G.C. 1968. Family Micropezidae, In: Papavero, N. (Ed.), A catalogue of the Diptera of the Americas south of the United States 48 . São Paulo, Departamento de Zoologia, Secretaria de Agricultura, 33 p.

Systema Dipterorum. 2013. The Biosystematic of World Diptera. Version 1.5. Available at: http://www.diptera.org (accessed 27 February 2014).

Received 13 November 2013; accepted 30 January 2014

Associate Editor: Marcia S. Couri 